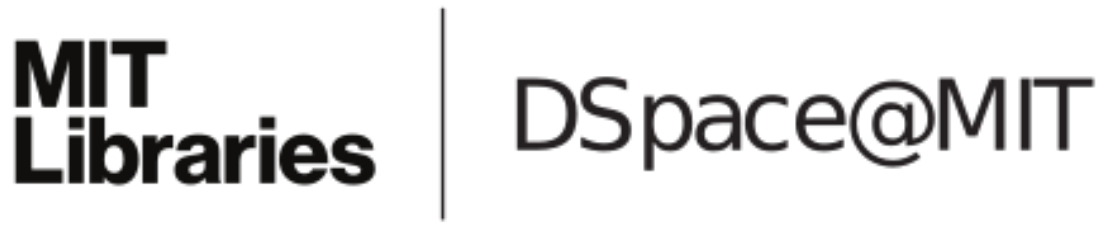

\author{
MIT Open Access Articles \\ Decoherence-free neutron interferometry
}

The MIT Faculty has made this article openly available. Please share how this access benefits you. Your story matters.

Citation: Pushin, D. A., M. Arif, and D. G. Cory. "Decoherence-free neutron interferometry." Physical Review A 79.5 (2009): 053635.(C) 2010 The American Physical Society.

As Published: http://dx.doi.org/10.1103/PhysRevA.79.053635

Publisher: American Physical Society

Persistent URL: http://hdl.handle.net/1721.1/51344

Version: Final published version: final published article, as it appeared in a journal, conference proceedings, or other formally published context

Terms of Use: Article is made available in accordance with the publisher's policy and may be subject to US copyright law. Please refer to the publisher's site for terms of use. 


\title{
Decoherence-free neutron interferometry
}

\author{
D. A. Pushin, ${ }^{1, *}$ M. Arif, ${ }^{2}$ and D. G. Cory ${ }^{1}$ \\ ${ }^{1}$ Massachusetts Institute of Technology, Cambridge, Massachusetts 02139, USA \\ ${ }^{2}$ National Institute of Standards and Technology, Gaithersburg, Maryland 20899, USA
}

(Received 10 September 2008; published 21 May 2009)

\begin{abstract}
Perfect single-crystal neutron interferometers are adversely sensitive to environmental disturbances, particularly mechanical vibrations. The sensitivity to vibrations results from the slow velocity of thermal neutrons and the long measurement time that are encountered in a typical experiment. Consequently, to achieve a good interference solutions for reducing vibration other than those normally used in optical experiments must be explored. Here we introduce a geometry for a neutron interferometer that is less sensitive to low-frequency vibrations. This design may be compared with both dynamical decoupling methods and decoherence-free subspaces that are described in quantum information processing. By removing the need for bulky vibration isolation setups, this design will make it easier to adopt neutron interferometry to a wide range of applications and increase its sensitivity.
\end{abstract}

DOI: 10.1103/PhysRevA.79.053635

PACS number(s): 03.75.Dg, 03.65.- w, 42.50.-p

\section{INTRODUCTION}

Neutron interferometry is one of the most precise techniques used to test the postulates of quantum mechanics, and it is also one of the most important and precise techniques used to measure low-energy neutron cross sections [1]. Although the fundamentals of neutron interferometry are easily recognizable from common optics, the slow velocity of neutrons $(1680 \mathrm{~m} / \mathrm{s}$ for the $2.35 \AA$ neutrons used in this study) and low count rates at the detector $(1000 / \mathrm{min})$ demand novel solutions. The most important of these was the development of multiblade single-crystal interferometery which enables high contrast to be observed with only limited beam alignment $[2,3]$. The challenge remains however of making the experiment robust against mechanical vibrations [4,5]. A typical single-crystal interferometer has path lengths of 10 $\mathrm{cm}$ with a typical $50 \mu$ s travel time of neutrons in the system. Small-amplitude low-frequency vibrations may significantly degrade the contrast. Here we propose a solution to robust interferometry based on a four-blade single-crystal geometry that reduces errors introduced by vibrations.

\section{INTERFEROMETER SCHEMATIC}

The most common three-blade geometry for a perfectcrystal neutron interferometer (NI) [1] is shown in Fig. 1(a) along with the four-blade configuration [Fig. 1(b)] that is less sensitive to low-frequency vibrations.

In the three-blade case the neutron beam, coming from the left, is coherently split into two paths by the first blade via Bragg scattering. After being reflected by the second blade, these two paths are recombined at the third blade. The resultant interference is observed at the $\mathrm{O}-$ and $\mathrm{H}$-detectors. Note that we align the $y$ coordinate parallel to the Bragg planes and the $x$ coordinate is perpendicular to the Bragg planes. In the four-blade case the situation is nearly identical with the significant difference that the paths are reflected and cross

\footnotetext{
*mitja@mit.edu
}

each other without interfering at the center of the interferometer; i.e., without a blade there is no mixing of the states.

It is sufficient to take a simple model for noise and to consider vibrations as sinusoidal oscillations around the center of mass of the single crystal, which we write as

\section{Three-blade single-crystal interferometer}
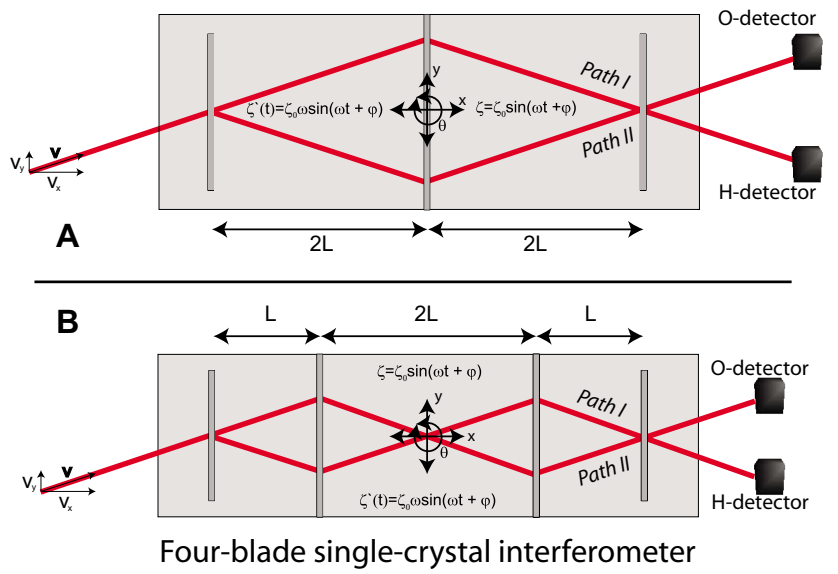

FIG. 1. (Color online) (a) A schematic diagram of the threeblade neutron interferometer. A neutron beam (with neutron velocity v) comes from the left, is split by the first blade, is diffracted on the second blade, and recombines at the third. After passing through the interferometer, the beam is captured by the O- and H-detectors. We model vibrations as oscillations of and around the center of mass of the interferometer, as $\zeta(t)=\zeta_{0} \sin (\omega t+\varphi)$, where $\zeta$ could be $y$ (transverse vibrations), $x$ (longitudinal vibrations), and $\theta$ (rotational vibrations). In order to compare oscillations between the three- and four-blade devices the distance between the blades is set equal to $2 L$. (b) A schematic diagram of the proposed interferometer with four blades. Instead of one diffracting blade here we have two, which reverses the neutron paths in order to compensate for vibrations. We will compare the performances using the same vibration modes with the same amplitudes. Note that for the three-blade interferometer the O-detector has the maximum contrast and in the four-blade interferometer the $\mathrm{H}$-detector has the maximum contrast. 


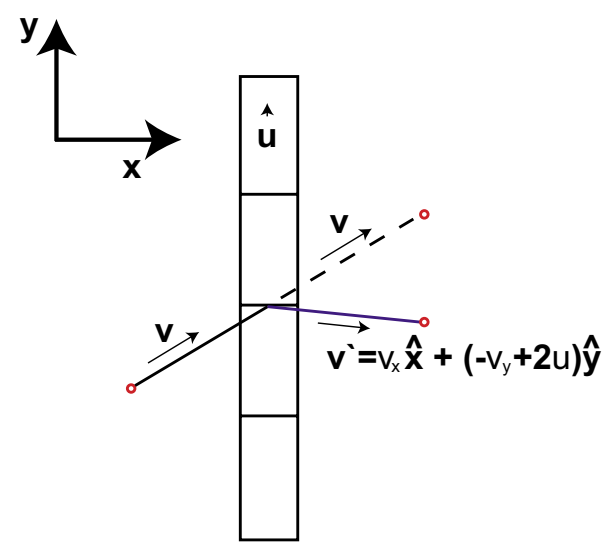

FIG. 2. (Color online) A schematic diagram of the neutron scattering from a blade of the neutron interferometer. Due to the crystal movement, the reflected neutron's momentum is modified. For the transmitted case, the momentum remains unaltered.

$$
\zeta(t)=\zeta_{0} \sin (\omega t+\varphi)
$$

For $\zeta$ we can specify any coordinates $x, y$, and $z$, or any angles (such as $\theta$, the rotation around $z$ axis). In order to motivate the discussion we adopt a simple model for the neutron-blade interaction that includes all of the necessary physics. The interaction is that of bouncing of a small particle (neutron) from a moving heavy wall (the blade) where the particle is reflected. When the particle is transmitted there is no interaction (Fig. 2). We use conservation of momentum and energy to calculate the neutron's change in velocity after bouncing. We require a small enough amplitude and low enough oscillation frequency that the modified momentum of the neutron still satisfies the Bragg condition.

Vibrations thus modify the neutron velocities and change the path length of the neutron inside the interferometer [6-9].

Using these approximations, it is clear that vibrations along every axis except the $z$ coordinate will reduce the interferometer contrast. The $z$ component of neutron velocity is zero and the path lengths are independent of crystal vibration along the $z$ axis at this level of approximation.

\section{VIBRATIONS ALONG THE $y$ AXIS}

We first consider vibrations along the $y$ axis. The measure of the quality of the interferometer is its contrast, so we calculate and plot the contrast versus the frequency of oscillations. The four-blade version returns higher contrast by compensating with the third blade for momentum change introduced by vibration of the second blade. We expect this compensation to be most precise for low-frequency vibrations.

\section{A. Three-blade interferometer}

Assume that the neutron hits the first blade of interferometer at the time $t=0$. We rewrite Eq. (1) for vibrations along the $y$ axis where $\varphi$ is a random phase between the arriving neutron and the vibrating blade,

$$
y(t)=y_{0} \sin (\omega t+\varphi) .
$$

The velocity of the interferometer at the time $t$ is

$$
u_{y}(t)=\frac{d y(t)}{d t}=y_{0} \omega \cos (\omega t+\varphi) .
$$

At time $t=0$, the velocity of the interferometer along the $y$ coordinate is $u_{y}(0)=y_{0} \omega \cos \varphi$. Conservation of momentum and energy at the moment $t=0$ implies that the velocity of the transmitted neutron does not change, while the reflected neutron's velocities are $\mathbf{v}_{\text {refl }}(0+)=v_{x} \hat{\mathbf{x}}-\left[v_{y}-2 u_{y}(0)\right] \hat{\mathbf{y}}$.

The phase difference for the neutron between path $I$ and path II is

$\Delta \Phi=\Phi($ path II $)-\Phi($ path I $)=\frac{1}{\hbar} \int_{\text {path II }} \mathbf{p} d \mathbf{s}-\frac{1}{\hbar} \int_{\text {path I }} \mathbf{p} d \mathbf{s}$,

where $\mathbf{p}$ is the momentum of the neutron and $\mathbf{s}$ is the pathlength vector along which the neutron is moving.

For the neutron to travel between the first two blades takes a time $t=2 L / v_{x}=2 \tau$. The contrast depends on the total phase difference between the paths. Notice that under these assumptions the two paths cross the third blade at the same spot and the travel time along these paths remains $4 \tau$. So, the loss in contrast seen in the presence of vibration is not due to the finite coherence length of the interferometer but rather is due to the extra phase shifts introduced by the vibrations.

Using these we find

$$
\Delta \Phi(\varphi)=16 \frac{m_{n}}{\hbar} \tau\left[v_{y}-u_{y}(0)\right]\left[u_{y}(2 \tau)-u_{y}(0)\right] .
$$

If we assume that $u_{y}(t)$ is slowly varying on the scale of $2 \tau$ (or $\omega \tau \ll 1)$, we can approximate the expression $u_{y}(2 \tau)-u_{y}(0)$ as a derivative of $u_{y}(t)$,

$$
\Delta \Phi(\varphi)=\left.16 \frac{m_{n}}{\hbar} \tau^{2}\left[v_{y}-u_{y}(0)\right] 2 \frac{d u_{y}(t)}{d t}\right|_{t=\tau} .
$$

The intensity at the $\mathrm{O}$ detector is

$$
I_{O}(\phi)=1+\cos [\Delta \Phi(\varphi)+\phi]
$$

and depends on the random phase $\varphi$. An experimental phase $\phi$ is introduced by a phase flag. We average the intensity over random phase $\varphi$ :

$$
\overline{I_{O}(\phi)}=\frac{1}{2 \pi} \int_{0}^{2 \pi}\{1+\cos [\Delta \Phi(\varphi)+\phi]\} d \varphi .
$$

The contrast $C$ as usual is defined as

$$
C=\frac{\max \{\overline{I(\phi)}\}-\min \{\overline{I(\phi)}\}}{\max \{\overline{I(\phi)}\}+\min \{\overline{I(\phi)}\}},
$$

where we vary the phase $\phi$ to find the max or min.

Fig. 3(a) shows the dependence of the contrast $C_{y}$ on the frequency of vibrations along the $y$ axis for the three-blade interferometer. The contrast was calculated for $L=5 \mathrm{~cm}$, a neutron velocity of $v=2000 \mathrm{~m} / \mathrm{s}$, and vibration amplitudes of $y_{0}=0.1 \mu \mathrm{m}$. Here we observe that the contrast starts to decrease near $100 \mathrm{~Hz}$. 

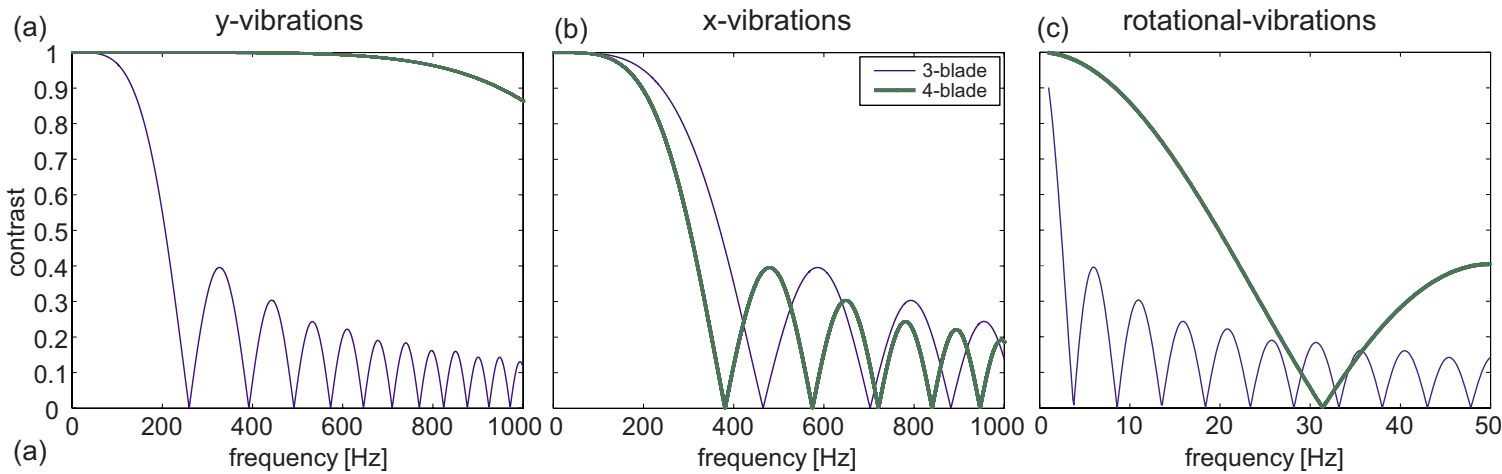

FIG. 3. (Color online) (a) Contrast due to vibrations along the $y$ axis. (b) Contrast due to vibrations along the $x$ axis. (c) Contrast due to rotational vibrations $\theta$ around $z$ axis and the center of mass. Note that the scale of the frequency axis in (c) is different from those shown in (a) and (b).

\section{B. Four-blade interferometer}

As in the three-blade interferometer case, we derive expressions for the phases the neutron acquires while traveling along the two interferometer paths. Here, the time for the neutron to travel between the first two blades is $\tau=L / v_{x}$. Note that the detector corresponding to the neutron paths with equal number of reflections for the three-blade interferometer is $\mathrm{O}$-detector and for the four-blade interferometer it is $\mathrm{H}$-detector.

Between the first blade and the second, the phases are identical to the three-blade interferometer case except that $2 L$ changes to $L$. The phase difference between path II and path $I$ is

$$
\Delta \Phi(\varphi)=8 \frac{m_{n}}{\hbar} \tau\left[u_{y}(0)-v_{y}\right]\left[2 u_{y}(0)-3 u_{y}(\tau)+u_{y}(3 \tau)\right]
$$

Again, as for the case of three-blade interferometer, we assume that the function $u_{y}(t)$ is slowly varying on the scale of $\tau$ (or $\omega \tau \ll 1$ ) and we rewrite the phase change in terms of a derivative,

$$
\begin{aligned}
\Delta \Phi(\varphi) & =16 \frac{m_{n}}{\hbar} \tau^{2}\left[v_{y}-u_{y}(0)\right]\left[\left.\frac{d u_{y}(t)}{d t}\right|_{t=\tau / 2}-\left.\frac{d u_{y}(t)}{d t}\right|_{t=2 \tau}\right] \\
& =\left.16 \frac{m_{n}}{\hbar} \tau^{3}\left[v_{y}-u_{y}(0)\right] \frac{3}{2} \frac{d u_{y}^{2}(t)}{d^{2} t}\right|_{t=5 \tau / 4} .
\end{aligned}
$$

Notice that the linear term drops out. This is the source of the protection against vibrations. The contrast comparison we make is the O-beam of the three-blade interferometer to the H-beam of the four-blade interferometer. The intensity at the $\mathrm{H}$-detector is

$$
I_{H}(\phi)=1+\cos [\Delta \Phi(\varphi)+\phi]
$$

and depends on the random phase $\varphi$ of vibration. Again we average the intensity over this random phase:

$$
\overline{I_{H}(\phi)}=\frac{1}{2 \pi} \int_{0}^{2 \pi}\{1+\cos [\Delta \Phi(\varphi)+\phi]\} d \varphi
$$

We obtain the contrast using Eq. (9). In Fig. 3(a) we plot the frequency dependence of the contrast for the four-blade interferometer. Notice that paths I and II cross the fourth blade at the same spot. From Fig. 3(a) we clearly see that the four-blade interferometer is predicted to be much less sensitive to $y$ vibrations.

\section{VIBRATIONS ALONG THE $x$ AXIS}

In the case of vibrations along the $x$ axis the momentum of the neutron is not modified (see Fig. 2). However the path length changes depending on the phase $\varphi$ of the oscillations at $t=0$.

Here the contrast is primarily limited by the neutron coherence length of $1 / \Delta k$. The four-blade geometry does not protect against this and indeed the influence of a finite coherence length is slightly worse due to the noise being introduced since the intervals between blades are changed by the vibrations. In the three-blade case there are two such intervals, while in the four-blade case there are three. So with finite $\Delta k$ the more blades there are, the worse the noise is. However, since the acceptance of the NI is small, $\Delta k$ is small and this contribution to the noise is small.

Here we calculate just the contribution to the loss in contrast from the phase shift introduced by vibrations along $x$ axis. The vibrations along the $x$ axis are

$$
x(t)=x_{0} \sin (\omega t+\varphi) .
$$

In this case the phase shift is due to the paths crossing at a point displaced from ideal as shown in Fig. 4. Once we find $\Delta x$ for each interferometer, then $\Delta l=2 \Delta x \tan \alpha \sin \alpha$ and the phase difference is

$$
\Delta \Phi(\varphi)=\frac{m_{n}}{\hbar} v \Delta l
$$

For the three-blade interferometer, we have $\tau=L / v_{x}$ and

$$
\Delta x \approx x(4 \tau)-2 x(2 \tau)+x(0)
$$

where we neglect distance $x(2 \tau)-x(0)(<1 \mu \mathrm{m})$ in comparison with $L(>1 \mathrm{~cm})$. For the four-blade interferometer 


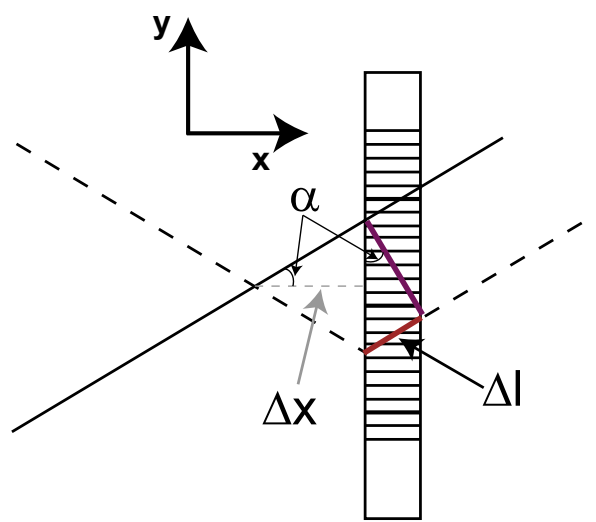

FIG. 4. (Color online) A schematic diagram of the neutron arriving to the third blade. Due to the crystal movement, the paths of the neutron will not recombine at the ideal point but at $\Delta x$ away from the third blade.

$$
\Delta x=x(4 \tau)-4 x(\tau)+3 x(0) .
$$

Using these $\Delta x$ we can get $\Delta \Phi$, substitute obtained $\Delta \Phi$ to find intensities, and average the intensities over different $\varphi$ to obtain the contrasts.

Figure 3(b) shows the contrast dependence on the frequency of vibrations along the $x$ axis. This should be viewed as an upper bound on the predicted contrast. The message is that vibrations along the $x$ axis are not very important even though the four-blade design is somewhat more sensitive to them than is the three blade design. In the final analysis the four-blade design is predicted to have better overall performance.

\section{ROTATION AROUND THE $z$ AXIS}

These vibrations are expected to be the most limiting since the neutron interferometer has such a small acceptance angle. In the case of rotational vibrations, we rewrite the oscillation in terms of the angle $\theta$ around the $z$ axis,

$$
\theta(t)=\theta_{0} \sin (\omega t+\varphi) .
$$

For small angles, rotational vibrations can be considered as translational vibrations, i.e., $\Delta r=r * \theta$, where $r$ is the distance from the blade to the center of rotation.

\section{A. Three-blade interferometer}

In the three-blade interferometer, the center of rotation is also the center of mass and the center of the middle blade. For the point [see Fig. 1(a)] where the neutron path crosses the blades, the rotational vibrations can be modeled as vibration along the $y$ axis for the first and last blades and along the $x$ axis for the path crossing the middle blade. In this case, the interaction with the middle blade does not change the velocity of the neutron. At the first blade we have a change in the momentum of the reflected beam and no change for the transmitted neutrons,

$$
\mathbf{v}_{\text {path I }}(t=0+)=v_{x} \hat{\mathbf{x}}+v_{y} \hat{\mathbf{y}},
$$

$$
\mathbf{v}_{\text {path II }}(t=0+)=v_{x} \hat{\mathbf{x}}+\left[-v_{y}+2 u 1_{y}(0)\right] \hat{\mathbf{y}},
$$

where $u 1_{y}(t)=2 L \theta_{0} \sin (\omega t+\varphi)$ velocity of the first blade in the $\hat{\mathbf{y}}$ direction.

The phase difference between the two paths is

$$
\begin{aligned}
\Delta \Phi(\varphi)= & \Phi(\text { path II })-\Phi(\text { path I })=\frac{m_{n}}{\hbar}\left[\left|\mathbf{v}_{\text {path I }}\right|^{2}\right. \\
& \left.-\left|\mathbf{v}_{\text {path II }}\right|^{2}\right] 4 \tau=8 \frac{m_{n}}{\hbar} L \theta_{0} \omega \sin \varphi\left[2 L \theta_{0} \omega \sin \varphi\right. \\
& \left.-v_{y}\right] 4 \tau,
\end{aligned}
$$

where $\tau=L / v_{x}$

Substituting this difference in phase into Eq. (7) for the O-beam intensity and averaging, we find the frequency dependence of contrast (9). Fig. 3(c) shows this contrast. As an amplitude of vibrations $\theta_{0}$ we used $1 \mu \mathrm{rad}$. The value for the amplitude comes from the current NIST setup, where a vibration isolation table is controlled to this level [5].

\section{B. Four-blade interferometer}

In the four-blade interferometer the center of rotation and the center of mass coincide between the blades. For points [see Fig. 1(b)] where the neutron path crosses the blades, the rotational vibrations are modeled as vibrations along the $y$ axis. As in the three-blade case the $v_{x}$ component of the neutron velocity does not change. The velocities are modified as follows.

For path I:

$$
\begin{gathered}
v_{y}(\mathrm{I}: 1 \rightarrow 2)=v_{y}, \\
v_{y}(\mathrm{I}: 2 \rightarrow 3)=-v_{y}+2 \sqrt{L^{2}+\left(v_{y} \tau\right)^{2}} \theta_{0} \omega \cos (\omega \tau+\varphi), \\
v_{y}(\mathrm{I}: 3 \rightarrow 4)=v_{y}-2 \sqrt{L^{2}+\left(v_{y} \tau\right)^{2}} \theta_{0} \omega[\cos (\omega \tau+\varphi) \\
+\cos (\omega 3 \tau+\varphi)],
\end{gathered}
$$

where $\tau=L / v_{x}$ and the sign of the last cosine term is positive because the oscillations of the second and the third blades have a $\pi$ phase-shift difference.

For path II:

$$
v_{y}(\mathrm{II}: 1 \rightarrow 2)=v_{y}+2 \theta_{0} \omega 2 L \cos \phi,
$$

$$
\begin{aligned}
v_{y}(\mathrm{II}: 2 \rightarrow & 3)=-v_{y}(\mathrm{II}: 1 \rightarrow 2)+2 \sqrt{L^{2}+\left(v_{y} \tau\right)^{2}} \theta_{0} \omega \cos (\omega \tau \\
& +\varphi), \\
v_{y}(\mathrm{II}: 3 \rightarrow & 4)=-v_{y}(\mathrm{II}: 2 \rightarrow 3)-2 \sqrt{L^{2}+\left(v_{y} \tau\right)^{2}} \theta_{0} \omega \cos (\omega 3 \tau \\
& +\varphi) .
\end{aligned}
$$

The phases along each path are as follows.

For path I:

$$
\Phi(\mathrm{I}: 1 \rightarrow 2)=\frac{m_{n}}{\hbar} v^{2} \tau,
$$




$$
\begin{gathered}
\Phi(\mathrm{I}: 2 \rightarrow 3)=\frac{m_{n}}{\hbar}\left[v_{x}^{2}+v_{y}(\mathrm{I}: 2 \rightarrow 3)^{2}\right] 2 \tau, \\
\Phi(\mathrm{I}: 2 \rightarrow 3)=\frac{m_{n}}{\hbar}\left[v_{x}^{2}+v_{y}(\mathrm{I}: 3 \rightarrow 4)^{2}\right] \tau .
\end{gathered}
$$

For path II:

$$
\begin{gathered}
\Phi(\mathrm{II}: 1 \rightarrow 2)=\frac{m_{n}}{\hbar}\left[v_{x}^{2}+v_{y}(\mathrm{II}: 1 \rightarrow 2)^{2}\right] \tau, \\
\Phi(\mathrm{II}: 2 \rightarrow 3)=\frac{m_{n}}{\hbar}\left[v_{x}^{2}+v_{y}(\mathrm{II}: 2 \rightarrow 3)^{2}\right] 2 \tau, \\
\Phi(\mathrm{II}: 2 \rightarrow 3)=\frac{m_{n}}{\hbar}\left[v_{x}^{2}+v_{y}(\mathrm{III}: 3 \rightarrow 4)^{2}\right] \tau .
\end{gathered}
$$

The phase difference is

$$
\begin{aligned}
\Delta \Phi(\varphi)= & \Phi(\mathrm{II}: 1 \rightarrow 2)+\Phi(\mathrm{II}: 2 \rightarrow 3)+\Phi(\mathrm{II}: 3 \rightarrow 4) \\
& -[\Phi(\mathrm{I}: 1 \rightarrow 2)+\Phi(\mathrm{I}: 2 \rightarrow 3)+\Phi(\mathrm{I}: 3 \rightarrow 4)] .
\end{aligned}
$$

As before, we can find the $I_{H}$ intensity at the $\mathrm{H}$ detector, average it over the random phase $\varphi$, and extract the contrast. This contrast dependence on the frequency of rotational vibrations is plotted in Fig. 3(c). We see that for these rotations the four-blade interferometer design is significantly more robust than the three-blade. Notice that for rotational vibrations the first moment of the loss of contrast is not equal to zero; all vibrations contribute to the loss of contrast.

\section{CONCLUSION}

Our model reconstructs the situation that is normally seen in neutron interferometry. Vibrations we use in our simulations (with amplitude of $10^{-7} \mathrm{~m}$ in translation) produce changes in the incident angle of the neutron of much less than the acceptance angle of the crystal $\left(\ll 5 \times 10^{-6} \mathrm{rad}\right)$ and of a similar order for the $50 \mathrm{~Hz}$ frequency range previously measured [5]. In order to exceed the acceptance angle the amplitude of vibrations would have to be bigger than $50 \mu \mathrm{m}$.

Note that small angle vibrations around the $x$ axis will be similar to the translational vibrations along the $y$ axis, and small angle vibrations around $y$ will be similar to the translational vibrations along $x$. As mentioned before, vibrations along the $z$ axis do not influence the contrast.

Taken together these results bring us to the four-blade experimental geometry for neutron interferometer. Although

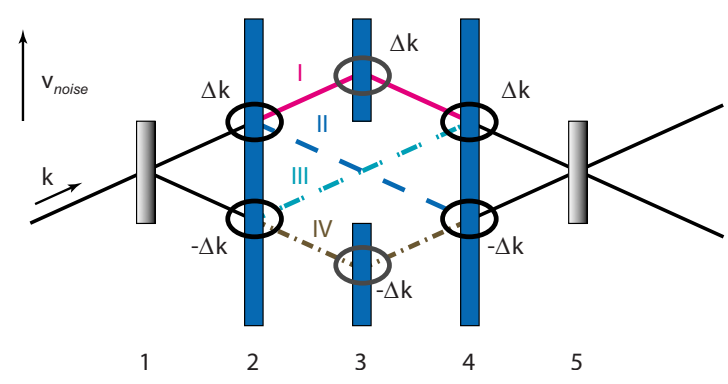

FIG. 5. (Color online) A schematic representation of the robust four-blade interferometer embedded in a five-blade interferometer. The overall interferometer operates over SU(4) and can be thought of as a tensor product space of two qubits. A simple abstraction of the noise generators is $\pm \Delta k$ at each internal reflection (there is no noise at the first and final blades). When we look over the entire path, two paths have noise (I and IV), and two paths do not have noise (II and III). The noise generator is proportional to $\operatorname{sgn}\left(\vec{k} \cdot \vec{v}_{\text {noise }}\right) \Delta k$ for each reflection internal to NI.

the four-blade design leads to a loss of half of the neutron intensity, we can make up for this loss with a more robust, small system that can be moved closer to the beam break. We thus regain and even increase the final neutron intensity at the detectors. In order to achieve high contrast the proposed four-blade interferometer system still requires excellent temperature stability [10].

The robust nature of the four-blade interferometer can also be understood as a result of dynamical decoupling or decoherence-free subspace (DFS). The dynamical decoupling analogy $[11,12]$ is easiest to see; the third set of blades acts to undo the change in momentum introduced by the second blades. Provided that the noise (motion of the NI) is the same when the neutron encounters both the second and third blades, then the momentum error is completely removed.

The DFS [13-15] picture can be seen if two additional paths are considered. Now we have four paths and an interferometer that acts over SU(4); see Fig. 5. The outer two paths are sensitive to the noise and the inner two are isolated from it. We are presently installing a five-blade interferometer to test these predictions.

\section{ACKNOWLEDGMENTS}

Support provided by NIST is gratefully acknowledged. The authors are grateful for discussions with D. L. Jacobson, R. Laflamme, B. Levi, and C. Ramanathan. Discussion with S. A. Werner about an earlier idea of a four-blade interferometer for use in gravity and spin-rotation [7] experiments is gratefully appreciated. 
[1] H. Rauch and S. A. Werner, Neutron Interferometry (Oxford University Press, New York, 2000).

[2] H. Rauch, W. Treimer, and U. Bonse, Phys. Lett. A 47, 369 (1974).

[3] W. Bauspiess, U. Bonse, H. Rauch, and W. Treimer, Z. Phys. 271, 177 (1974).

[4] A. Shimony and H. Feshbach, Physics As Natural Philosophy (MIT Press, Cambridge, MA, 1987).

[5] M. Arif, D. E. Brown, G. L. Greene, R. Clothier, and K. Littrell, Proc. SPIE 2264, 20 (1994).

[6] C. G. Shull and N. S. Gingrich, J. Appl. Phys. 35, 678 (1964).

[7] B. Buras and T. Giebultowicz, Acta Crystallogr., Sect. A:
Cryst. Phys., Diffr., Theor. Gen. Crystallogr. 28, 151 (1972). [8] M. Dresden and C. N. Yang, Phys. Rev. D 20, 1846 (1979).

[9] B. Mashhoon, Phys. Rev. Lett. 61, 2639 (1988).

[10] D. A. Pushin, M. Arif, M. G. Huber, and D. G. Cory, Phys. Rev. Lett. 100, 250404 (2008).

[11] L. Viola and S. Lloyd, Phys. Rev. A 58, 2733 (1998).

[12] L. Viola, E. Knill, and S. Lloyd, Phys. Rev. Lett. 82, 2417 (1999).

[13] P. Zanardi and M. Rasetti, Phys. Rev. Lett. 79, 3306 (1997).

[14] L.-M. Duan and G.-C. Guo, Phys. Rev. Lett. 79, 1953 (1997).

[15] D. A. Lidar, I. L. Chuang and K. B. Whaley, Phys. Rev. Lett. 81, 2594 (1998). 